\title{
Low frequency of moaA3 gene among the clinical isolates of Mycobacterium tuberculosis from Tamil Nadu and Pondicherry - south eastern coastal states of India
}

\author{
Balaraman Sekar*1, Kamalanathan Arunagiri ${ }^{1}$, Nagamiah Selvakumar ${ }^{2}$, \\ Kaluvuri Serena Preethi ${ }^{1}$ and Kandhaswami Menaka ${ }^{1}$
}

Address: ${ }^{1}$ Division of Laboratories, Central Leprosy Teaching and Research Institute, Chengalpattu, Tamil Nadu, India and ${ }^{2}$ Department of Mycobacteriology, Tuberculosis Research Centre, Chennai, Tamil Nadu, India

Email: Balaraman Sekar* - drbsekar@yahoo.com; Kamalanathan Arunagiri - aruna_giri@rediffmail.com; Nagamiah Selvakumar - selvakumar.nagamiah@gmail.com; Kaluvuri Serena Preethi - serenapreethi@gmail.com; Kandhaswami Menaka - kmenaka.2008@rediffmail.com

* Corresponding author

Published: 25 July 2009

BMC Infectious Diseases 2009, 9:1 I4 doi:10.1 186/147|-2334-9-1/4

This article is available from: http://www.biomedcentral.com/I47/-2334/9/114

(c) 2009 Sekar et al; licensee BioMed Central Ltd.

This is an Open Access article distributed under the terms of the Creative Commons Attribution License (http://creativecommons.org/licenses/by/2.0), which permits unrestricted use, distribution, and reproduction in any medium, provided the original work is properly cited.
Received: 18 December 2008

Accepted: 25 July 2009

\begin{abstract}
Background: Comparative genomic analysis of $M$. tuberculosis H37Rv and $M$. bovis BCG have shown that 16 RDs (Regions of Differences) are deleted in BCG and have shown six deletion regions in M. tuberculosis H37Rv. RDI, is present in M. tuberculosis but is absent in all M. bovis BCG sub-strains. A study from Kerala, a south-western coastal state of India aimed to find out differences in RDI region showed for the first time the presence of moaA3 gene in majority of their clinical isolates, that was absent in type strain $\mathrm{H} 37 \mathrm{Rv}$. We attempted to find out such polymorphism between type strains and the clinical isolates within RDI, targeting moaA3 gene among the clinical isolates of Tamil Nadu \& Pondicherry, south-eastern coastal states of India
\end{abstract}

Methods: One hundred and sixteen clinical isolates of $M$. tuberculosis were included in the study. PCR using RDIDLa and RDIDRa primers was carried out to amplify a 652 bp fragment, encoding for $c f p 10$ and esat 6 proteins of RDI. A second PCR using primers designed from the surrounding regions of moaA3 gene was done to confirm the presence of the full Open Reading Frame (ORF) in clinical isolates.

Results: In M. tuberculosis H37Rv the expected 652 bp band was present. In BCG it was absent as expected, but a 386 bp fragment was amplified. Around 12/116 (10.3\%) of our clinical isolates showed both 652 and 386 bp fragments. The additional $386 \mathrm{bp}$ amplicon is a part of the moaA3 gene which codes for molybdopterin cofactor protein A in M. bovis. The second PCR amplified the flanking sequence of moaA3 and yielded the expected amplicon of $1254 \mathrm{bp}$ in all those $10.3 \%$ of clinical isolates which had the 386 bp fragment. However the earlier study carried out in Kerala, reported the presence of moaA3 gene in majority $(97 \%)$ of their clinical isolates.

Conclusion: This finding showed that there was regional variation presenting polymorphism in moA3 gene, among the strains of $M$. tuberculosis and further strengthens the speculation of genetic differences among the strains of Kerala and Tamil Nadu \& Pondicherry, the South Indian states 


\section{Background}

The passaging of M. tuberculosis $\mathrm{H} 37 \mathrm{Rv}$ and M. bovis BCG over years brought out changes in their genomic and virulence characteristics. Mahairas et al described the genomic changes in Region of difference 1 (RD1) - a region that is present in all virulent laboratory and clinical strains of Mycobacterium bovis and Mycobacterium tuberculosis [1]. The loss of RD1 in M. bovis BCG, contributes to the attenuation and its reintroduction into an attenuated strain resulted in a significant increase in virulence [2]. Deletions of RvD1 to RvD5 and TbD1 in H37 Rv are also reported [3].

A study was planned previously to screen the clinical isolates of M. tuberculosis from Kerala, a south-western costal state of South India for differences in RD1 region [4]. Amplification using primers that span the ORF coding for cfp10 and esat 6 has shown to have produced 652 bp in all M. tuberculosis clinical isolates studied and also an extra amplicon of 386 bp was amplified in $97 \%$ of local field isolates, but not in M. tuberculosis H37 Rv. Further analysis revealed that the primer that was made spanning the RD1 region was similar to the portions of the moaA3 gene in the RvD5 region, which resulted in the amplification of 386 bp fragment. This amplicon spanned the nucleotides 575 to 960 of the moaA3 gene in M. bovis

This prompted us to screen the local isolates from states of Tamil Nadu and Pondicherry, on the south-eastern coastal region of South India for the presence of moaA3 gene. Our study revealed a low frequency of moaA3 gene among Tamil Nadu and Pondicherry clinical isolates compared to higher frequency of moaA3 among the clinical isolates from the neighbouring Kerala state.

\section{Methods \\ Mycobacterial Strains}

In this study, we included 116 M.tuberculosis non-repeat clinical isolates from different parts of Tamil Nadu and Pondicherry. (74 - Obtained from Tuberculosis Research Centre (TRC), Chennai, Tamil Nadu and 42 from Government Chest Disease Hospital, Pondicherry). All the strains included in this study were isolated in the year 2006 and the analysis was completed by April 2007. M. tuberculosis H37RV and M.bovis BCG were used as type strains. All the strains were identified by biochemical analysis.

Since this study was based on laboratory work carried out with mycobacterial strains and there was no experimental work involving human (or) animal, the ethical approval was not required.

\section{Drug susceptibility Testing (DST)}

The in-vitro DST was carried out on solid LowensteinJensen (LJ) medium using the Minimal Inhibitory Con- centration (MIC) method/Resistance ratio (RR) method [5].

Briefly a $3 \mathrm{~mm}$ loopful of the bacterial suspension containing approximately $4 \mathrm{mg} / \mathrm{ml}$ was used to inoculate drug-free and drug-containing slopes (32, 64 and $128 \mathrm{mg} /$ $\mathrm{ml}$ ). The standard strain, $M$. tuberculosis $\mathrm{H} 37 \mathrm{Rv}$ was included in every batch of tests as a check on the inoculumn size as well as the drug concentrations in the medium. The slopes were incubated at $37^{\circ} \mathrm{C}$ and read at the end of 28 days. The lowest drug concentration which inhibited growth (defined as 20 colonies) was taken as the MIC. RR was defined as the ratio of the MIC of the test isolate to the MIC of the reference strain $\mathrm{H} 37 \mathrm{Rv}$.

\section{Extraction of DNA from clinical samples}

All the samples were processed for DNA extraction as per the standard method of Herman et al [6]. Essentially, the cells were lysed with lysozyme followed by treatment with proteinase $\mathrm{K}$ and sodium dodecyl sulphate. Proteins and macromolecules were precipitated using $\mathrm{NaCl}$ and hexadecyltrimethylammonium bromide $-\mathrm{NaCl}$ solutions. Nucleic acids were recovered from aqueous phase after extraction with chloroform and isoamyl alcohol. DNA was further precipitated 30 minutes with isopropanol at $20^{\circ} \mathrm{C}$. The pellet was washed with ethanol and later reconstituted in TE buffer.

\section{$P C R$ amplification of $R D I$ region}

PCR amplification of the RD1 was carried out as described earlier [4]. Briefly Primers RD1 DLa (For):-5'-AGA TGA AGA CCG ATG CCG CTA C-3', RD1 DRa (Rev):-5'-CCC GTG TTT CGC TAT TCT ACG C-3' that can span the RD1 region Rv3874 and Rv3875 coding for $c f p 10$ and esat 6 were used. After initial denaturation, amplification was performed in PCR thermocycler (Minicycler-MJ Research) for 35 cycles at $95^{\circ} \mathrm{C} / 40 \mathrm{sec}, 64^{\circ} \mathrm{C} / 1 \mathrm{~min}, 72^{\circ} \mathrm{C} / 1 \mathrm{~min}$ followed by a final extension at $72^{\circ} \mathrm{C} / 5 \mathrm{~min}$. It was expected to yield a 652 bp product and also 386 bp products if moaA3 was present in the test isolates, only $652 \mathrm{bp}$ product in M. tuberculosis H37Rv and only 386 bp products in M. bovis BCG.

To identify the flanking sequence of the 386 bp another set of primers were used (moaFP - 5'-CCC ATC GTG GTC GTT CAC C-3' and moaRP - 5'-CGA TGG CAG CGG TTT ACA G-3') to amplify a 1254 bp product, with the same conditions as done for the above amplification.

\section{PCR amplification for IS6 I IO}

Amplification of DNA for IS6110 was performed with primers IS-F - 5'-CCTGCGAGCGTA GG CGTCGG-3'and IS-R - 5'CTCGTCCAGCGCCGCTTCGG-3', to amplify 123 bp fragment of insertion element IS6110 of M. tuberculosis complex as reported earlier [7]. Briefly PCR was carried 
out in $50 \mu \mathrm{l}$ volume, using $200 \mu \mathrm{M}$ dNTPs, 20 pM of each primer and $1 \mathrm{U}$ of Taq polymerase, followed by template. Conditions followed were initial denaturation at $94^{\circ} \mathrm{C}$ for 4 minutes, followed by 35 cycles at $90^{\circ} \mathrm{C}$ for 1 minute, $60^{\circ} \mathrm{C}$ for 1 minute, and $72^{\circ} \mathrm{C}$ for 1 minute, and a final extension at $72^{\circ} \mathrm{C}$ for 10 minutes.

\section{Results \\ Screening of RDI}

The PCR primers designed to amplify regions within RD1 to find out the polymorphism between the type strain and the clinical isolates amplified the expected $652 \mathrm{bp}$ fragment (comprising of Rv 3874 and Rv 3875, coding for cfp10 and esat 6) in M. tuberculosis H37Rv. In M.bovis BCG, the 652 bp was absent as expected, but a 386 bp fragment was amplified. All the 116 clinical isolates showed $652 \mathrm{bp}$ products. However in 8 out of $74(10.8 \%)$ of Tamil Nadu isolates and 4 out of 42 (9.5\%) of Pondicherry isolates showed both 652 and 386 bp fragments (Figure 1). Thus, 12 out of the $116(10.3 \%)$ samples showed the extra amplicon of $386 \mathrm{bp}$. (Table 1)

The second PCR using primers designed from the surrounding region of moaA3 gene done to confirm the presence of the full ORF in clinical isolates, amplified the expected $1254 \mathrm{bp}$ in all the clinical isolates which showed the extra 386 bp amplicon. (Figure 2) (Table 2)

As our isolates showed a lower frequency of moaA3 gene, we checked the possibility of false negativity by including 50 DNA templates from those isolates which were positive for 652 bp of RD1, but found negative for 386 bp extra amplicon and ran second PCR to amplify the flanking sequences. None of these templates were positive for flanking sequence. Thus, the remote possibility of false negativity as a cause for lower frequency of moaA3 gene among our isolates was ruled out.

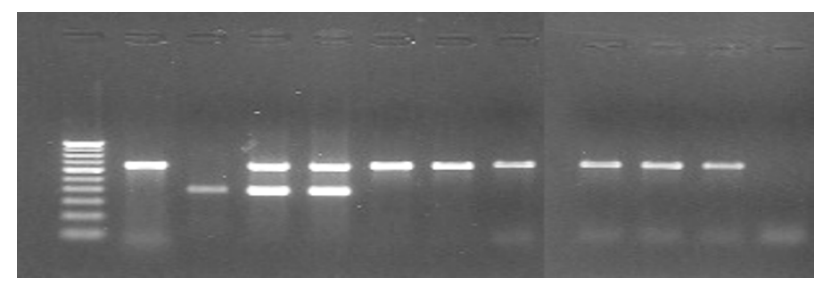

\section{Figure I}

PCR for amplification of cfp 10 and esat 6 of RD I.

Lane-I - Molecular weight marker (I00 bp ladder). Lane-2 M. tuberculosis H37Rv, showing only 652 bp (RDI). Lane-3 $M$. bovis BCG showing only 386 bp. Lane-4 \& 5 - Clinical isolates showing 652 bp \& 386 bp extra amplicon (moaA3).

Lane-6 to II - Clinical isolates showing 652 bp only. Lane I 2 - Negative control.

\section{Screening of IS 6110}

PCR for IS6110 showed the 123 bp fragment in all 116 (100\%) clinical isolates. (Table 1)

\section{Correlation with drug susceptibility results}

For 58 TRC clinical isolates, drug susceptibility result was available. Resistance to Streptomycin (S), Isoniazid (H), Rifampicin(R), and Ethambutol (E) were 33\%, 14\%, 0\% and 25\%, among moaA3 positive samples where as among moaA3 negative samples were 29\%, 34\%, 20\% and 14\% respectively. Multi Drug Resistance (MDR) was observed in $18 \%(9 / 46)$ of samples negative for moaA3, where as no MDR was observed among moaA3 positive sample. However, as most of the samples tested were moaA3 negative and only few samples were positive the statistical significance could not be drawn for want of adequate sample size in both the comparable groups.

\section{Discussion}

Studies with subtractive hybridization and microarrays have identified 16 regions present in $M$. tuberculosis H37Rv but are absent in M. bovis BCG $[1,8]$. Further deletions of RvD1 to RvD5 and TbD1 genes in H37 Rv are also reported [3]. RD1 region comprises of nine genes ( $\mathrm{Rv} 3871$ to $\mathrm{Rv} 3879 \mathrm{c}$ ) and spans a $9.5-\mathrm{kb}$ region. In $\mathrm{M}$. bovis BCG, RD1 deletion completely removes seven genes (Rv3872 to Rv3878) and truncates two others (Rv3871 and Rv3879c) [9].

Of the nine genes predicted within the $9.5 \mathrm{~kb} \mathrm{RD} 1$ region, those coding for $c f p 10(\operatorname{Rv} 3874)$ and esat $6(\mathrm{Rv} 3875)$ are immunogenic and RD1 deletion mutants of M. tuberculosis have been found to be less virulent [9]

Thus, generation of deletion of genes appear to be a major mechanism for creating genetic diversity.

Based on this, a study from Kerala was carried-out to screen for differences in the RD1 region. Amplification using primers that span the ORF coding for $c f p 10$ and esat 6 was expected to give a 652 bp PCR product. But the PCR results revealed an extra amplicon of 386 bp in $97 \%$ of the clinical isolates screened. Further characterization by sequencing and homology search indicated that this region is a part of the moaA3 gene which encodes for molybdopterin cofactor protein A in M.bovis. The PCR primer that was made spanning the RD1 region was shown to be similar to portions of the moaA3 (MT3427) gene in the RvD5 region in clinical isolates and also in CDC1551 which resulted in the amplification of the 386 bp fragment. This amplicon spanned the nucleotides 575 to 960 of the moaA3 gene (MT 3355) in M. bovis [4]

With this background, we searched for the presence of moaA3 gene among the clinical isolates collected from 
Table I: PCR for amplification of $c f p / 0$ and esat 6 of RD I

\begin{tabular}{|c|c|c|c|c|}
\hline Source of samples & No. of samples investigated & $\begin{array}{l}\text { No. of samples positive for } \\
\text { IS6110 }\end{array}$ & $\begin{array}{l}\text { No. of samples positive for } \\
652 \text { bp }\end{array}$ & $\begin{array}{c}\text { No. of samples positive for } 652 \\
\text { bp }+386 \text { bp }\end{array}$ \\
\hline Tamil Nadu & 74 & $74(100 \%)$ & $74(100 \%)$ & $08(10.8 \%)$ \\
\hline Pondicherry & 42 & 42 (100\%) & $42(100 \%)$ & $04(9.5 \%)$ \\
\hline Total & 116 & $116(100 \%)$ & $116(100 \%)$ & $12(10.3 \%)$ \\
\hline
\end{tabular}

$652 \mathrm{bp}$ - product found in M. tuberculosis H37Rv; $386 \mathrm{bp}$ - product found in M. bovis BCG

Tamil Nadu and Pondicherry. Our search showed presence of 652 bp of $c f p 10$ and esat 6 of RD1 region in all the 116 clinical isolates screened but only a limited number of $12 / 116(10.3 \%)$ clinical isolates showed the presence of $386 \mathrm{bp}$ amplicon of moaA3. Amplification of flanking sequence of $386 \mathrm{bp}$ moaA3 showed the expected $1254 \mathrm{bp}$ product in among all the isolates showed the extra $386 \mathrm{bp}$ amplicon. Thus, in contrast to Kerala study, only in limited proportion of our isolates moaA3 was amplified using the designed primer.

Although Rao et al, [10] reported total absence of RD1 region in clinical isolates, in a study from Hyderabad, Andhrapradesh (a south eastern coastal state) all our clinical isolates showed the presence of RD1 (cfp 10 and east 6) region. In fact, few isolates we obtained from Hyderabad were found positive for RD1 region in all the isolates (data not shown). This is in concurrence with other reports $[11,4]$.

In H37Rv, the moaA3 amplicon was shown to be absent. The RvD5 region from which the amplicon was generated is an IS6110 mediated deletion in the type strain H37Rv [3]. IS6110 is a powerful genetic marker for strain differentiation [12]. In general low/no copies of IS6110 were

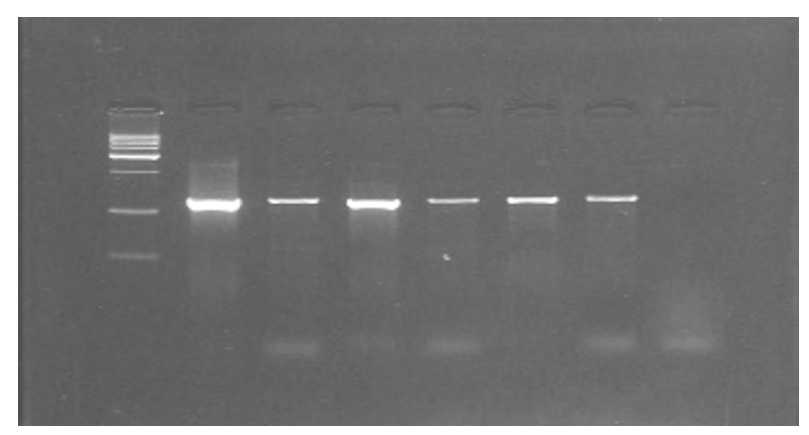

\section{Figure 2}

PCR for amplification of flanking sequence of $386 \mathrm{bp}$ of moaA3 gene. Lane-I - Molecular weight marker (500 bp ladder). Lane-2 to 7 - Clinical isolates which were positive for 386 bp extra amplicon, showing I254 bp of flanking sequence. Lane-8 - Negative control. implicated among south-east Asian strains, including India [13-15].

However, a closer scrutiny at the frequency of no/low copies of IS6110 reveals that they are commoner among Kerala strains than those from Tamil Nadu. An analysis from Kerala [16] reported about $24 \%$ of no copies and $39 \%$ of single copy of IS6110 in their isolates. Thus, around $62.5 \%$ of (50 out of 80 ) strains analyzed were not typeable by IS6110 based finger printing. This reported to be higher than the small numbers of IS6110 - deficient strains $-1 \%-4 \%$ reported in Tamil Nadu $[17,18]$. It is appropriate to underscore that all our isolates screened for IS6110 by PCR were found positive.

Adding to this genetic variations, our finding of low frequency of $10.3 \%$ of moaA3 gene among Tamil Nadu and Pondicherry isolates compared to the higher proportion of $97 \%$ among Kerala isolates, further strengthens the speculation of genetic variation among the strains of Kerala and Tamil Nadu \& Pondicherry, the South Indian states.

Further in screening of different genes of RD1 region among clinical isolates from Kerala and Western and Northern India, $\operatorname{Rv} 3871$ and $\mathrm{Rv} 3872$ (part of RD1 region) was reported as high as $98 \%$ in Kerala isolates, where as only $30 \%$ was reported in the other isolates screened [11].

This further emphasizes the need to carry out systematic molecular epidemiological studies in these endemic areas to explore any other genetic variations. Further the role of IS6110 or any other insertion sequences or mobile genetic elements in the genetic variation may also be investigated.

\section{Conclusion}

Our attempt to screen for moaA3 gene among the clinical isolates of Tamil Nadu and Pondicherry revealed a low frequency of moaA3 gene compared to a high frequency of moaA3 gene among Kerala isolates. This finding showed a regional variation presenting polymorphism in moA3 gene, among the strains of M.tuberculosis and further strengthens the speculation of genetic variations among 
Table 2: PCR for amplification of Flanking sequence of 386 bp of moaA3 gene

\begin{tabular}{lccc}
\hline Source of samples & No. of samples investigated & No. of samples positive for 652 bp +386 bp & $\begin{array}{c}\text { No. of samples positive for Flanking sequence } \\
\text { among samples showing } 386 \text { bp amplicon }\end{array}$ \\
\hline Tamil Nadu & 74 & $08(10.8 \%)$ & $08(100 \%)$ \\
Pondicherry & 42 & $04(9.5 \%)$ & $04(100 \%)$ \\
Total & 116 & $12(10.3 \%)$ & $12(100 \%)$ \\
\hline
\end{tabular}

386 bp product found in $M$. bovis BCG \& in some field isolates

1254 bp product coded flanking sequence of $386 \mathrm{bp}$ fragment of moaA3 gene

the strains of Kerala and Tamil Nadu \& Pondicherry, the neighbouring South Indian states.

\section{List of abbreviations}

RD: Region of Difference; BCG: Bacille Calmette-Guerin; cfp 10: culture filtrate protein 10; esat 6 : early secreted antigenic target 6; ORF: Open Reading Frame; PCR: Polymerase Chain Reaction; MIC: Minimal Inhibitory Concentration; MDR: Multi Drug Resistance; DST: Drug susceptibility Testing.

\section{Competing interests}

The authors declare that they have no competing interests.

\section{Authors' contributions}

BS conceived, designed and supervised the study and carried out data analysis and wrote the manuscript. KA COdesigned the study and carried out most of the experiment and assisted in the data analysis and preparation of the manuscript. NSK participated in the design and coordination of the study. KSP did part of the experimental work and coordinated. KM co-participated in most of the experiments and assisted in the data analysis

\section{Acknowledgements}

We thank Government Chest Disease Hospital, Pondicherry and Tuberculosis Research Centre, Chennai for the supply of their clinical isolates and we thank Dr. P. K. Oommen, Director, Central Leprosy Teaching \& Research Institute, for his permission and support.

\section{References}

I. Mahairas GG, Sabo PJ, Hickey MJ, Singh DC, Stover CK: Molecular analysis of genetic differences between Mycobacterium bovis BCG and virulent M. bovis. J Bacteriol 1996, I 78(5): I 274- I 282.

2. Pym AS, Brodin P, Brosch R, Huerre M, Cole ST: Loss of RDI contributed to the attenuation of the live tuberculosis vaccines Mycobacterium bovis BCG and Mycobacterium microti. Mol Microbiol 2002, 46(3):709-717.

3. Brosch R, Philip W], Stavropoulos E, Colston MJ, Cole ST, Gordon SV: Genomic analysis reveals variation between Mycobacterium tuberculosis $\mathrm{H} 37 \mathrm{Rv}$ and the attenuated $\mathrm{M}$. tuberculosis H37Ra strain. Infect Immun 1999, 67( I I):5768-5774.

4. Sarojini S, Soman S, Radhakrishnan I, Mundayoor S: Identification of MoaA3 gene in patient isolates of Mycobacterium tuberculosis in Kerala, which is absent in M. tuberculosis $H 37 R v$ and H37 Ra. BMC Infect Dis 2005, 5:8I.

5. Canetti G, Fox W, Khomenko A, Mahler HT, Menon NK, Mitchison DA, et al: Advances in techniques of testing mycobacterial drug sensitivity and use of sensitivity tests in tuberculosis control programmes. Bull World Health Organ 1969, 4I:2 I-43.

6. Hermans PW, Schuitema AR, Van Soolingen D, Verstynen CP, Bik EM, Thole JE, Kolk AH, van Embden JD: Specific detection of Mycobacterium tuberculosis complex strains by Polymerase Chain reaction. J Clin Microbiol 1990, 28(6): | 204-12 13.

7. Sekar B, Selvaraj L, Allexis A, Ravi S, Arunagiri K, Rathinavel L: The Utility of IS6I I 0 sequence based Polymerase Chain Reaction in comparison to conventional methods in the diagnosis of Extra-pulmonary tuberculosis. Indian J Med Microbiol 2008, 26(4):352-355.

8. Behr MA, Wilson MA, Gill WP, Salamon H, Schoolnik GK, Rane S, Small PM: Comparative genomics of BCG vaccines by wholegenome DNA microarray. Science 1999, 284(54 I 9): I520- 1523.

9. Lewis KN, Liao R, Guinn KM, Hickey MJ, Smith S, Behr MA, Sherman DR: Deletion of RDI from Mycobacterium tuberculosis mimics Bacille Calmette-Guerin attenuation. J Infect Dis 2003, 187(1): I 17-123.

10. Rao KR, Kauser F, Srinivas S, Zanetti S, Sechi LA, Ahmed N, Hasnain $\mathrm{SE}$ : Analysis of genomic downsizing on the basis of region of difference polymorphism profiling of Mycobacterium tuberculosis patient isolates reveals geographic partitioning. J Clin Microbiol 2005, 43(1 2):5978-5982.

II. Soman S, Joseph BV, Sarojini S, Kumar RA, Katoch VM, Mundayoor S: Presence of Region of Difference I among clinical isolates of Mycobacterium tuberculosis from India. J Clin Microbiol 2007, 45(10):3480-348I.

12. van Soolingen D, Hermans PW, de Hass PE, Scoll DR, van Embden JD: Occurrence and stability of insertion sequences in Mycobacterium tuberculosis complex strains: evaluation of an insertion sequence-dependent DNA polymorphism as a tool in the epidemiology of tuberculosis. J Clin Microbiol 1991, 29(I I):2578-2586.

13. Fomunkong NG, Tang TH, al-maamary S, Ibrahim WA, Ramayah S, Yates M, Zainuddin ZF, Dale JW: Insertion sequence typing of Mycobacterium tuberculosis: characterization of a widespread subtype with a single copy of IS6II 0 . Tuber Lung Dis 1994, 75(6):435-440.

14. Palittapongarnpim P, Luangsook $\mathrm{P}$, Tansuphaswadikul S, Chuchottaworn C, Prachaktam R, Sathapatayavongs B: Restriction fragment length polymorphism study of Mycobacterium tuberculosis in Thailand using IS6IIO as probe. Int J Tuberc Lung Dis 1997, I (4):370-376.

15. Yuen LKW, Ross BS, Jackson KM, Dwyer B: Characterization of Mycobacterium tuberculosis strains from Vietnamese patients by Southern Blot hybridization. J Clin Microbiol 1993, 31(6): $1615-1618$

16. Radhakrishnan I, Manju YK, Kumar RA, Mundayoor S: Implication of low frequency of IS6 I I 0 in fingerprinting field isolates of Mycobacterium tuberculosis from Kerala, India. J Clin Microbiol 200I, 39(4): 1683.

17. Narayanan S, Das S, Garg R, Hari L, Rao VB, Frieden TR, Narayanan PR: Molecular epidemiology of Tuberculosis in rural area of high prevalence in South India: Implication for disease control and prevention. J Clin Microbiol 2002, 40( I 2):4785-4788.

18. Das S, Paramasivan CN, Lowrie DB, Prabhakar R, Narayanan PR: IS6I I 0 restriction fragment length polymorphism typing of clinical isolates of Mycobacterium tuberculosis from patients with pulmonary tuberculosis in Madras, South India. Tuber Lung Dis 1995, 76(6):550-554. 


\section{Pre-publication history}

The pre-publication history for this paper can be accessed here:

http://www.biomedcentral.com/1471-2334/9/114/pre

pub

Publish with Bio Med Central and every scientist can read your work free of charge

"BioMed Central will be the most significant development for disseminating the results of biomedical research in our lifetime. " Sir Paul Nurse, Cancer Research UK

Your research papers will be:

- available free of charge to the entire biomedical community

- peer reviewed and published immediately upon acceptance

- cited in PubMed and archived on PubMed Central

- yours - you keep the copyright 